\title{
DEL PRECLÁSICO MEDIO \\ AL CLÁSICO TEMPRANO: UNA PROPUESTA \\ DE FECHAMIENTO PARA EL ÁREA NUCLEAR \\ DE IZAMAL, YUCATÁN ${ }^{1}$
}

\author{
Lucía Quiñones Cetina \\ Universidad Autónoma de Yucatán
}

Izamal se encuentra localizado en la parte media de las planicies del norte de la península de Yucatán, un área ubicada entre la costa norte y las colinas del sur de la península.

El sitio se caracteriza por edificios de estilo megalítico y por un sistema de sacbeoob que, aparentemente, lo unían con asentamientos de menor rango, probablemente bajo su dominio.

Izamal tenía un área de aproximadamente $10 \mathrm{~km}^{2}$. La parte central del sitio aún conserva restos de los imponentes edificios que se encontraban distribuidos en torno a plazas. La principal era de cerca de $300 \mathrm{~m}$ de largo por $200 \mathrm{~m}$ de ancho; en sus costados oriente y poniente se levantaban templos dedicados a Itzamná, el ltzamatul y el Kabul; al norte estaba el Kinich Kak Moo, dedicado al dios solar, y al sur se ubicaba el Ppap Hol Chac ${ }^{2}$ (Millet y Burgos, 2001).

En 1992 inicia el Proyecto Izamal, dirigido por el arqueólogo Luis Millet Cámara, cuyos objetivos han sido restaurar los principales edificios prehispánicos, comprender la evolución sociocultural de Izamal y las relaciones que mantuvo con ciudades circunvecinas, y analizar el material arqueológico, incluida la cerámica.

El análisis del material cerámico, basado en los atributos perceptibles a simple vista, tales como el acabado de superficie, formas de las vasijas, decoración y pasta — características con significado temporal y espacial—, permite establecer las dimensiones de tiempo y espacio de los vestigios arqueológicos y de la gente que los fabricó y usó. Asimismo, refleja evoluciones culturales y aproximaciones a modelos de organización socioeconómica e ideológica (Forsyth, 1983: 5; Varela, 1994: 96).

\footnotetext{
${ }^{1}$ Este trabajo es un resumen de mi tesis de licenciatura, que lleva el mismo título, agradezco a la arqueóloga Sylviane Boucher su asesoría.

${ }^{2}$ El Habuc, uno de los tres edificios del área nuclear de Izamal que se han intervenido, se ubica al sureste de la Plaza Principal, entre las calles 26 y 28 por 35 y 37 de la población actual (Quiñones, 2003: 22).
} 
El sistema de clasificación y análisis que se utilizó en este trabajo fue el de Tipo-Variedad, apoyado en atributos observables a simple vista que se organizan en categorías taxonómicas (vajilla, grupo, tipo, variedad), en combinación con una clasificación analítica que utiliza atributos modales y su distribución para reflejar tradiciones y horizontes estilísticos, de tal manera que la combinación de esos dos análisis produzca información relativa a cronología, distribuciones cerámicas e interrelaciones que permitan identificar procesos culturales (Boucher y Azul, 2001).

El sistema Tipo-Variedad se compone de dos niveles de integración:

1. En el primero se realiza un examen de los atributos de la cerámica (pasta, construcción, forma, técnicas de decoración o diseño) que se combinan en la formación de un artefacto y son observables empíricamente. Como resultado de este estudio la cerámica se describe y ordena en las categorías básicas: vajilla, grupo, tipo y variedades (Willey, Culbert y Adams, 1967: 304; Ball, 1978: 3).

2. El segundo nivel de análisis permite establecer hipótesis y teorías, así como relaciones cerámicas entre varios sitios a escala regional, a través de conceptos abstractos de integración, como los complejos cerámicos, horizontes cerámicos, esferas cerámicas, etc. (Willey, Culbert y Adams, op. cit: 305, 306; Robles, 1990: 28).

Para definir los complejos cerámicos de Izamal se procedió tradicionalmente, ordenando los tipos con base en su filiación con los complejos ya establecidos para sitios de la Planicie del Norte así como con otros ${ }^{3}$ cuyas colecciones pueden ser consultadas en la Ceramoteca del Centro Regional INAH Yucatán.

Aunque estamos conscientes de que la ordenación cronológica basada en la filiación de los tipos cerámicos con los "complejos-esfera" —establecidos por Smith en 1971, Andrews, en 1989, y otros para la región del norte de Yucatán-, representa un procedimiento simplista de fechamiento tipológico que puede no tener la validez necesaria para el establecimiento de secuencias cronológicas (ver Boucher y Dzul, op. cit.), este método propone una evaluación cronológica relativa que, por supuesto, deja abierta la posibilidad de otras interpretaciones.

Con base en el resultado del análisis de los 68527 tiestos recuperados en las excavaciones de Izamal, se propone el establecimiento de nueve complejos cerámicos, de los cuales se analizan cuatro: Izquierdo, Kahlo, Ureta y Varo.

${ }^{3}$ Mayapán (Smith,1971); Dzibilchaltún (Simmons, 1980); Komchén (Andrews V, 1989); Tzubil (Boucher,1991); Oxkintok (Varela, op. cit.); Mérida (rescates: Costa del Sol (Pool, 1997) y Salvador Alvarado (Azul, 2001)); Chichen Itzá (Pérez de Heredia, 1998), Xcambó (Jiménez, 2002 y Ceballos, 2003); Sayil (Boucher y Palomo, s/f); Cobá (Robles, op. cit.); Xelha (Canché, 1992), y San Gervasio (Peraza, 1993), entre otros. 
Está integrado por cinco grupos cerámicos: Sabán, Ucú, Juventud, Dzudzuquil y Muxanal, y representa el $0.1 \%$ del total del material analizado.

Es el material más temprano localizado en Izamal y guarda similitud con el complejo Nabanché Temprano de Komchén, pues comparten los grupos Dzudzuquil y Juventud, así como los grupos que Andrews $V$ denominó Achiotes y Chunhinta, y a los cuales se decidió nombrar Sabán y Ucú, respectivamente, retomando la propuesta de Smith (op. cit.: 31,32 ), quien considera local la producción de ambos grupos.

Los grupos Juventud y Ucú están presentes tanto en el Preclásico Medio como en el Preclásico Tardío (Andrews, op. cit.: 4, 19), mientras que el grupo Sabán, con sus tipos Sabán Burdo: variedad Sabán, y Chancenote Estriado: variedad Chancenote, representa una de las tradiciones cerámicas más perdurables de la secuencia de Izamal, pues está presente hasta el Clásico Temprano, lo cual demostraría el carácter conservador de la población izamaleña prehispánica con respecto a las vajillas cerámicas de uso doméstico.

Por su parte, los grupos Dzudzuquil y Muxanal, aunque escasamente representados, son materiales diagnósticos del Preclásico Medio (Andrews, op. cit.: $22,24)$. El grupo Muxanal, según considera este autor (op. cit.: 33), podría haber sido importado desde algunos sitios del sur del área maya, pues muestra similitud con el Muxanal que se reporta en Becán. Empero, consideramos que el grupo Muxanal podría ser una producción local que copia modas del Petén, dadas las diferencias que se observan entre los ejemplares de este grupo de Izamal y Dzibilchaltún con los de Becán y Calakmul. ${ }^{4}$

Considerando el carácter local de los grupos Ucú y Sabán, y las diferencias entre los grupos Muxanal del área maya central y los de las planicies del norte, creemos que los tipos cerámicos del complejo Izquierdo de Izamal representan desarrollos locales con una marcada influencia de tradiciones Mamón del horizonte del Preclásico Medio del Petén. Asimismo, probablemente integren una esfera cerámica temprana de las tierras bajas mayas del norte, ya que estos grupos están presentes en diversos sitios del área, como Komchén, Acanceh, Ek Balam, Yaxuná, Oxkintok y Tzubil, entre otros.

\footnotetext{
${ }^{4}$ Las diferencias entre ambos grupos estriban en el acabado de superficie y en la pasta. Los tiestos procedentes de Becán y Calakmul se caracterizan por un engobe crema opaco, mientras que en los de Dzibilchaltún e Izamal destaca el acabado lustroso de textura cerosa del engobe crema. Por otra parte, la pasta del grupo Muxanal de Becán es de textura mediana, compacta, de color rojo claro a café grisáceo, con desgrasante de calcita y frecuentes inclusiones rojas. Los de Izamal, en cambio, presentan una pasta roja de textura fina, compacta, con partículas pequeñas de calcita y granos rojos como desgrasante.
} 


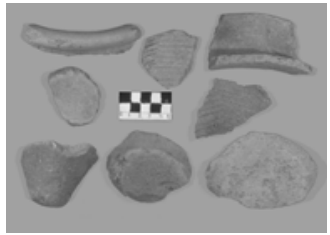

Foto 1. El grupo SABán está presente en la tradición cerámica de lzamal desde el Preclásico hasta el Clásico Temprano

Foto 1A. El grupo Ucú podría ser una producción local inspirada en un grupo similar del área maya central, denominado Chunhinta

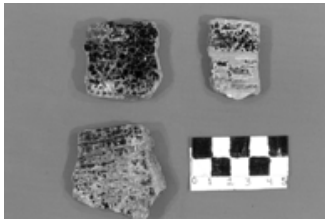

\section{Complejo Kahlo (Preclásico Tardío, 450-150 a.C.)}

Está conformado por ocho grupos: Sabán, Achiote, Sierra, Juventud, PolveroSierra, Ucú, Flor y Carolina, los cuales representan el $1.06 \%$ del total del material analizado.

La presencia de los grupos Ucú y Juventud muestra una continuidad de las tradiciones cerámicas del Preclásico Medio (700-450 a.C.), aunque en el complejo Kahlo se observa una mayor diversidad en la forma de las vasijas, entre las que se incluyen formas asociadas con ritos ceremoniales, como las vasijas en forma de hongo del grupo Sierra (Boucher, comunicación personal, 2001).

El grupo cerámico mejor representado en el complejo Kahlo es el Sierra, el cual es un marcador diagnóstico para el horizonte Chicanel o Preclásico Superior (450-150 a.C.) (Ball, 1978: 81). El grupo Sierra del complejo Kahlo está estrechamente relacionado con su análogo del complejo Nabanché Tardío de Komchén; presentan las mismas características de acabado de superficie, pasta y formas de vasijas. Andrews (op. cit.: 5) considera que este grupo podría ser parte de una tradición local de monocromos rojos que se inicia en el Preclásico Medio (700-450 a.C.) con el grupo Juventud y concluye en el Protoclásico (150 a.C.-250 d.C.) con el grupo Xanabá. 
Dentro de la vajilla sin engobe de uso doméstico predomina, hasta el Clásico Temprano, el grupo Sabán de producción local, sin embargo en el complejo Kahlo se incluye, aunque en menor proporción, el grupo Achiotes, miembro de la vajilla Uaxactún sin engobe, tradición originaria del área maya central (Forsyth, op. cit.: 60).

Por su parte, el grupo Flor posiblemente sea un producto de importación del área maya central (Forsyth, op. cit.: 51), ya que ha sido reportado en varios sitios de las planicies del norte aunque en pequeños porcentajes (Ball, op. cit.: 86).

El grupo Carolina, reportado con mayor frecuencia en los sitios de la costa nordeste de Yucatán y en la oriental del norte de Quintana Roo, se presume como una expresión cerámica propia de las costas nororientales de la península de Yucatán (Peraza, op. cit.: 69), y comienza a manifestarse, aparentemente, desde el Preclásico Superior (450-150 a.C.), ya que su pasta y el acabado ceroso que presenta su engobe son parecidos a los del grupo Sierra.

Durante el complejo Kahlo, Izamal participa de las relaciones regionales de las planicies del norte, en donde son comunes los grupos Sabán, Sierra, Ucú y Juventud, pero al mismo tiempo comienza a establecer conexiones con el área maya central y con sitios de la costa nororiental de la península de Yucatán, como lo muestra la presencia de ollas domésticas del grupo Achiote, ollas y cajetes de vajillas de servicio con engobe monocromo crema del grupo Flor, acaso de importación, así como ollas y cajetes con doble engobe que posiblemente caractericen el final del período Preclásico Superior e inicio del Protoclásico en Izamal.

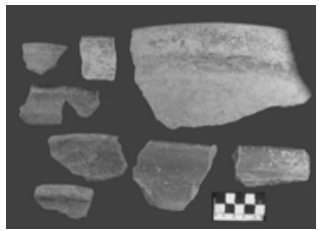

Foto 2. El grupo Sierra es el mejor representado en el complejo Kahlo, y es un marcador diagnóstico del horizonte Chicanel del Preclásico Superior

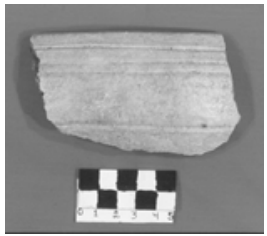

Foto 2A. El grupo Flor es posiblemente un producto de importación del área maya central. En la imagen se observa un fragmento de un cajete del tipo Acordeón Inciso de ese grupo 
Este complejo está integrado por los grupos Xanabá, Sierra, Polvero, Unto y 4 grupos no designados, que representan el $12 \%$ del total del material. También forman parte del complejo Ureta los grupos Sabán, Carolina y Huachinango, que continúan hasta el Clásico Temprano.

El complejo Ureta está circunscrito al horizonte cerámico Protoclásico, el cual se puede definir como un estado de desarrollo general entre el Preclásico y el Clásico (Brady et al., 1998: 17), ya que significa la presencia de un conjunto específico de modas cerámicas que marca una espectacular reorientación en las vigentes durante el Preclásico, y son un presagio de las características de acabado de superficie, decoración, diseños y formas de vasijas que predominarán en el Clásico Temprano (Forsyth, 1989: 54).

Los cambios más obvios son el surgimiento de un engobe anaranjado en lugar del rojo que imperó en el Preclásico, así como una relativa uniformidad de las formas de vasijas en contraste con la diversidad de formas que se observó en el Preclásico (Forsyth, op. cit.: 55).

Entre los atributos cerámicos diagnósticos asociados al Protoclásico se cuentan: un engobe blanco o crema debajo de un engobe naranja lustroso, soportes mamiformes y la decoración al negativo de la vajilla Usulután, propia de El Salvador, o su imitación, que es lo que con frecuencia se encuentra en las tierras bajas mayas, más que verdaderos ejemplares de esa vajilla (Brady et al., op. cit.: 22). La presencia de los tipos Iberia Naranja del grupo Águila e Ixcanrío Anaranjado Policromo, caracterizados por grandes soportes mamiformes -ambos integrantes de la esfera cerámica Floral Park que se manifiesta en las tierras bajas mayas del sur- así como de tiestos que parecen ser imitación del tipo Ixcanrío originario de Centroamerica, fortalecen la definición del complejo Ureta de Izamal y su inclusión dentro del Protoclásico.

El tipo Iberia Naranja representa numerosos experimentos locales para producir un engobe rojo claro o naranja con acabado brilloso, que es la característica principal del período Clásico (Brady, et al., op. cit.: 32). En lzamal se observó que a pesar de la similitud en cuanto a formas que este tipo presenta con su análogo de la secuencia cerámica de Calakmul (cajón C-25-11 CRY INAH), el material tiene una pasta más amarillenta y un engobe anaranjado, mientras que en los ejemplos de Calakmul presentan una pasta rosada y su engobe tiende a ser más rojizo y con acabado brilloso, por lo que es probable que el lberia Naranja de Izamal sea una producción local.

El tipo Ixcanrío Anaranjado Policromo no había sido reportado en las planicies del norte. En Izamal se encontraron 15 tiestos que habían sido clasificados en un principio como Dos Arroyos Anaranjado Policromo y Timucuy Anaranjado Policromo, sin embargo, por rasgos modales como los soportes mamiformes de sus vasijas y el doble engobe que presentaban algunos tiestos, se reclasificaron como tipo Ixcanrío Anaranjado Policromo, en el que se identificaron dos pastas: 
una de color rojo y textura fina, que habían sido incluidos en el tipo Dos Arroyos Anaranjado Policromo y otra de color amarillo rojizo con textura mediana, clasificada en un principio como Timucuy Anaranjado Policromo; los tiestos de la segunda pasta no presentaban la técnica de doble engobe, por lo que se clasificaron como "otro grupo no designado imitación Ixcanrío", ya que tiene las mismas formas de vasijas y los mismos diseños decorativos que el tipo lxcanrío Anaranjado Policromo. Por lo tanto, consideramos que el "otro grupo no designado imitación Ixcanrío" podría ser una producción local inspirada por la presencia del tipo Ixcanrío Anaranjado Policromo.

Asimismo, se encontraron tiestos decorados con la técnica al negativo, que evocan a la vajilla Usulután, pero con una pasta semejante a la del grupo Xanabá del complejo Ureta de Izamal, por lo que se catalogaron como "no especificado imitación Usulután", que podrían representar otra imitación de producción local.

Uno de los grupos cerámicos mejor representados que integran el complejo Ureta de Izamal es el grupo Xanabá, sin duda una manifestación propia de las planicies noroccidentales de Yucatán, ya que en esta región aparece con mucha frecuencia y gran variedad de formas y técnicas decorativas (Canché, op. cit.: 73).

El tipo Shangurro Rojo sobre Naranja, cuyas formas principales son cajetes con ángulo basal, fue establecido por Ball (op. cit.: 110), quien lo incluye dentro de un grupo con el mismo nombre, aunque presenta características similares al grupo Xanabá en cuanto a color y textura del engobe, pasta y formas de vasijas, por lo que consideramos que pertenece a este último grupo. Por otra parte, Ana Shepard advierte que la pasta y desgrasante de Shangurro Rojo sobre naranja son similares a los del tipo Valladolid Bícromo Inciso (Simmons, op. cit.: 221) que Smith (op. cit.: 31) había incluido dentro del grupo Timucuy. No obstante, en el presente estudio se han situado ambos, Shangurro Rojo sobre Naranja y Valladolid Bícromo Inciso, dentro del grupo Xanabá, puesto que su pasta y engobe están más relacionados con éste que con Timucuy (Sylviane Boucher, comunicación personal, 1998).

El grupo Polvero generalmente se sitúa en el Preclásico Tardío (450 a.C.-250 d.C.), sin embargo, seguimos la propuesta de Andrews (op. cit.: 8), quien lo ubica dentro del complejo Xculul del Protoclásico en Komchén, además de que argumenta la similitud que existe entre este grupo y el Xanabá del mismo complejo, en cuanto a pastas y acabados de superficie.

En el complejo Ureta se incluyeron las variedades Escamosa y Bordes Rojos del tipo Sierra Rojo del grupo Sierra. Ambas variedades fueron establecidas por Andrews V (op. cit.: 10-11) en la secuencia cerámica de Komchén. La variedad Escamosa la ubica en el complejo Xculul ya que considera que presenta un engobe con características similares al de Xanabá. Por otra parte, la forma principal de las vasijas de la variedad Bordes Rojos de Izamal, cajetes con ángulos redondeados en $Z$, se relaciona, usualmente, con tipos propios del Clásico Temprano (Forsyth, 1983: 64). 
Estas variedades del tipo Sierra Rojo podrían ser manifestaciones propias de inicios del Clásico Temprano, en las que aún se usan recetas de pastas del Preclásico Tardío, pero con formas y decoración bícroma características del Clásico Temprano.

Por último, el grupo Huachinango es considerado como una expresión característica de las costas del norte y oriente de Yucatán (Robles, op. cit:: 82), aunque probablemente su área de producción estuviese en la región cercana a Ek Balam (Bey y Bond, 1996). Cronológicamente ha sido ubicado desde el Preclásico Terminal (Ball, op. cit.: 110) hasta el Clásico Temprano (Peraza, op. cit.: 64).

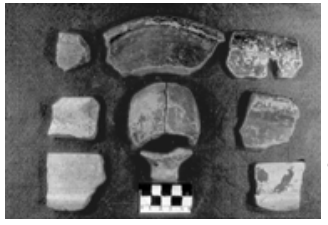

Foto 3. El grupo Sanaba es una manifestación propia de las planicies noroccidentales de Yucatán, donde se encuentra con mucha frecuencia y gran variedad de formas y técnicas decorativas

Foto 3a. El. TIPo Shangurro Rojo sobre Naranja había sido incluido dentro de un grupo del mismo nombre. Sin embargo, por las características de su pasta y engobe lo hemos incluido en el grupo Xanabá

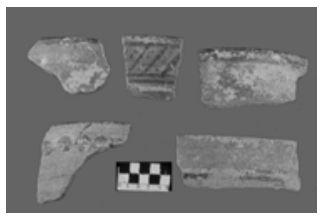

\section{Complejo Varo (Clásico Temprano, 250-600 d.C.)}

Está conformado por ocho grupos cerámicos: Sabán, Triunfo, Águila, Balanza, Alex, Carolina, Huachinango y Maxcanú, cuyas muestras conforman el $17.02 \%$ del total del material analizado.

Las ollas sin engobe del grupo Sabán están presentes desde el Preclásico, y en el Clásico Temprano se manifiestan a través de las variedades Becoob, del tipo Sabán sin Engobe, y Chiquilá, del tipo Chancenote Estriado.

Dentro de la tradición cerámica de las planicies del norte la policromía empieza a surgir probablemente desde el Preclásico Superior y Protoclásico, tal vez 
como resultado de la imitación de vasijas policromas importadas, como es el caso de Otro Grupo Imitación Ixcanrío del Protoclásico. Este tipo posiblemente sea precursor del tipo Timucuy Anaranjado Policromo, con quien comparte texturas y color de pasta.

En el presente estudio se estableció el tipo monocromo Alex Anaranjado que, en cuanto a color y texturas de pasta así como acabado de superficie, guarda estrechas analogías con el tipo Timucuy Anaranjado Policromo; además, respecto a forma y tamaño, es similar a las ollas de la variedad Chac del tipo Timucuy. Por tanto, con base en los atributos físicos de ambos tipos se decidió incluirlos como componentes del grupo Alex, siguiendo el mismo procedimiento metodológico que Forsyth (1983: 66; 1989: 9) usó para incluir al tipo Dos Arroyos Anaranjado Policromo en el grupo Águila.

Las ollas y cazuelas del grupo Maxcanú parecen haber sido populares en el occidente de la península durante el Clásico Temprano y el inicio del Clásico Tardío (Varela, op. cit.: 122). El tipo Zi Negro sobre Ante, de este grupo, posiblemente forme parte de una tradición decorativa con pintura negra chorreada que es común en las planicies del norte desde el Preclásico Superior hasta el Postclásico. Por su parte la variedad impresa del tipo Zi presenta analogías modales con el tipo Lakín Impreso del Clásico Tardío.

El grupo Huachinango se ha reportado con mayor frecuencia en sitios costeros de Quintana Roo y en el interior, centro y oriente del estado de Yucatán (Peraza, op. cit.: 64), pero es en Ek Balam en donde se ha encontrado el mayor número, lo cual podría indicar que este sitio está cerca de, o bien es el centro de distribución de este grupo cerámico (Bey y Bond, op. cit.). Aunque es en Izamal donde se ha hallado mayor variabilidad de formas, además de los cajetes con paredes rectodivergentes, se han identificado ollas, cajetes de silueta compuesta y una forma parecida a la del tipo Hongo Compuesto del grupo Sierra.

Los grupos Águila y Balanza son considerados como marcadores diagnósticos de la esfera Tzakol. Lo cual, junto con la presencia de los grandes cajetes de reborde basal de los tipos Dos Arroyos Anaranjado Policromo y Caldero Bayo Policromo, hace pensar en probables relaciones de intercambio entre Izamal y las zonas del Petén o área maya central.

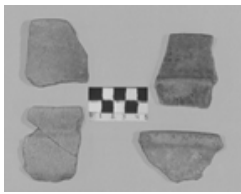

Foto 4A. ÁguILA NARANJA 


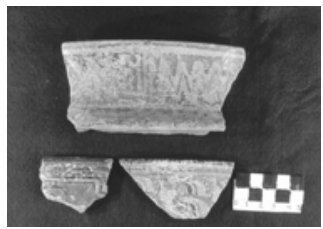

Foto 4B.

Dos Arroyos

NARANJA

Polícromo

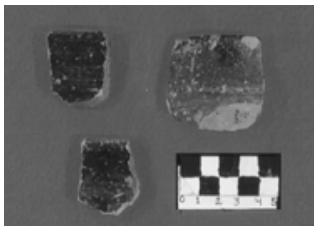

Foто $4 \mathrm{C}$.

Bal.anza Negro

Los grupos Águila (a, b) y Balanza (c) son considerados marcadores diagnósticos de la esfera Tzakol del área maya central. Su presencia en Izamal podría representar relaciones de intercambio entre ambas zonas

\section{Conclusiones}

Dado que uno de los objetivos de este estudio es el fechamiento del área central de Izamal, con base en las evidencias cerámica y arquitectónica se propone la siguiente cronología relativa para los edificios hasta ahora intervenidos de esta zona.

Kinich Kak Moo. La construcción de este edificio se propone entre el período Protoclásico (150 a.C.-250 d.C.) y el Clásico Temprano (250-600 d.C.). En el Postclásico Tardío (1200-1520 d.C.), el Kinich Kak Moo es objeto de una modificación estructural que ocurrió al fallar la resistencia de las piedras que soportan la moldura en delantal, las cuales descansaban sobre un muro vertical, por lo que fue necesario reforzarlas en todo el perímetro del basamento con un muro en talud (Millet y Burgos, op. cit.) 


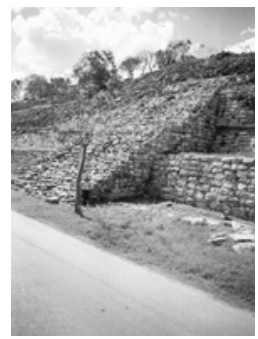

Foтo 5. Kinıch KaK Moo. Aspecto de la escalinata y la moldura en delantal del costado este de la Gran Plataforma

Itzamatul. Consideramos que el primer período constructivo del Itzamatul se realizó a fines del Protoclásico (150 a.C.-250 d.C.) o principios del Clásico Temprano (250-600 d.C.); la segunda modificación pudo haber tenido lugar en el Clásico Terminal (800-1000 d.C.) y el tercer período constructivo probablemente se llevó a cabo a finales del Clásico Terminal o principios del Postclásico Temprano (1000-1200 d.C.) (Quiñones, en Mollet et al., mecanografiado, 1998).

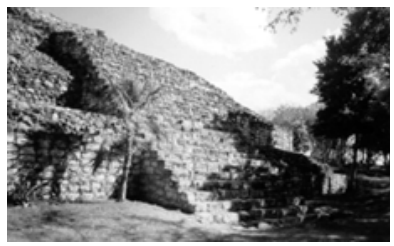

Foto 6. Itzamatul. Se reconocieron tres períodos constructivos: el primero corresponde a una pirámide de $20 \mathrm{~m}$ de altura con muros en talud. En el segundo, los muros en talud fueron cubiertos por muros verticales y esquinas en ángulo recto. La tercera modificación corresponde a la construcción de una gran plataforma de $6 \mathrm{~m}$ de altura que rodea al edificio. En la imagen se aprecian aspectos del segundo período constructivo 
Habuk. La primera etapa constructiva del Habuk puede fecharse para el Clásico Temprano (250-600 d.C.), y la segunda, durante el Clásico Terminal (8001000).

En cuanto a las filiaciones cerámicas de Izamal se puede considerar que para el Preclásico Medio (700-450 a.C.) o complejo Izquierdo, Izamal estaba inmerso en la esfera Nabanche (Temprana), de clara tradición de las planicies del norte, en tanto que en el siguiente complejo Kahlo o Preclásico Superior (450-150 a.C.), empieza a perfilarse cerámica doméstica y de servicio no sólo de la esfera $\mathrm{Na}$ banche (Tardía), sino también de la esfera Chicanel, relacionada con el área maya central, demostrando así su participación en la conocida homogenización cerámica entre las tierras bajas mayas del sur y del norte.

Durante el Protoclásico o complejo Ureta, los grupos cerámicos corresponden, en su mayoría, a la esfera Xculul de las tierras bajas del norte, así como a grupos cerámicos afiliados a la esfera Floral Park de la región de Belice. En el Clásico Temprano o complejo Varo, los grupos cerámicos pertenecen, mayormente, a la esfera local Cochuah, aunque también están presentes grupos cerámicos correspondientes a la esfera Tzakol, relacionada con el Petén guatemalteco o área maya central.

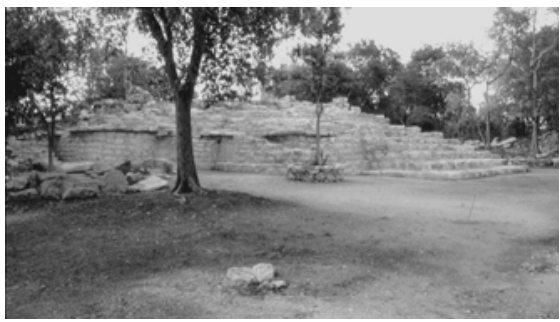

Foто 7. El. Habuk es una estructura formada por una plataforma de $90 \mathrm{~m}$ por lado y $3.80 \mathrm{~m}$ de altura, sobre la que se encuentra una plaza de $30 \mathrm{~m}$ de largo por 25 de ancho, delimitada por cuatro edificios. En la imagen se observa al edificio 1, situado en el costado poniente 


\section{BIBLIOGRAFÍA}

Andrews V., Willey

1988-1989 "Ceramic units from Komchén, Yucatán, México", Cerámica de Cultura Maya (15): 51-64, Filadelfia, Pennsylvania: Temple University.

Ball, Joseph

1978 "Archaeological pottery of the Yucatan-Campeche Coast", Studies in the Archaeological of Coastal Yucatán and Campeche. Middle American Research Institute (46): 69-146, Nueva Orleáns: Tulane University.

Bey, George, Tara Bond, W. Ringle, G. Hanson, C. Houck y C. Peraza Lope 1996 "The ceramic chronology of Ek Balam, Yuc. Mex.", Ancient Mesoamerica, 1(9): 101-120. Cambridge: Cambridge University Press.

Boucher Sylviane

1991 Informe de la cerámica de excavación en Tzubil, Yuc. Mecanografiado. México: Archivo INAH.

- y Sara Dzul

2001 La secuencia tipológica preliminar de la cerámica del Proyecto Arqueológico Calakmul, Campeche (temporadas 1993-2000), ponencia presentada en el Congreso Internacional de Cultura Maya. Yucatán, México.

Boucher, Sylviane y Yoli Palomo

(s.f.) Informe de la cerámica de excavación en Sayil, Yuc. (temporada 1987-1988). Mecanografiado. México: Archivo INAH.

Brady, J., J. Ball, Ronald Bishop, D. Pring, N. Hammond y R. Housley

1998 "The lowland maya 'Protoclassic' a reconsideration of its nature and significance", Ancient Mesoamerica, 1(9): 17-38. Cambridge: Cambridge University Press.

Canché Manzanero, Elena

1992 "La secuencia cerámica de Séla, Quintana Roo". Tesis de licenciatura en Ciencias Antropológicas con especialidad en Arqueología, México: Universidad Autónoma de Yucatán.

Ceballos Gallareta, Teresa y Socorro Jiménez

2000 "La esfera cerámica Cochuah-Chikín de las comarcas prehispánicas de Izamal y Tho para el Clásico Temprano (250-600 d.C.)”, Ichcansiho (5): 8-9. Mérida: Ayuntamiento de Mérida.

Ceballos Gallareta, Teresa

2003 "La cronología cerámica del puerto maya de Xcambó, costa norte de Yucatán: complejo Xtampú”. Tesis de licenciatura en Ciencias Antropológicas con especialidad en Arqueología. México: Universidad Autónoma de Yucatán. 
Dzul Góngora, Sara

2001 "Salvamento arqueológico en la colonia 'Salvador Alvarado Oriente' de Mérida, Yuc.". Tesis de licenciatura en Ciencias Antropológicas con especialidad en Arqueología. México: Universidad Autónoma de Yucatán.

Forsyth, Donald

1983 Investigation at Edzná, Campeche, Mex. Provo, Utah: Brigham University (Papers of the New World Archaeological Foundation, 46).

1989 The ceramics of El Mirador, Petén, Guatemala. N.W.A.F. Provo, Utah: Brigham Young University (El Mirador Series. Part 4).

Jiménez, Socorro

2002 "La cronología cerámica del puerto maya de Xcambó, costa norte de Yucatán: complejo Xcambó". Tesis de licenciatura en Ciencias Antropológicas con especialidad en Arqueología. México: Universidad Autónoma de Yucatán.

Millet Cámara, Luis et al.

1995 Informe del Proyecto Arqueológico Izamal, Yucatán. Temporada 1994-1995. Mecanografiado. México: Archivo Centro INAH.

Millet Cámara, Luis et al.

1996-1998 Informe del Proyecto Arqueológico Izamal, Yucatán. Temporada 19961998. Mecanografiado. México: Archivo Centro INAH.

Millet Cámara, Luis y Rafael Burgos

2001 "Izamal: una aproximación a su arquitectura", ponencia presentada en el I Congreso Internacional de Cultura Maya. Mérida, Yucatán.

Peraza Lope, Carlos

1993 "Estudio y secuencia del material cerámico de San Gervasio, Cozumel". Tesis de licenciatura en Ciencias Antropológicas con especialidad en Arqueología, México: Universidad Autónoma de Yucatán.

Pérez de Heredia Puente, Eduardo

1998 La cerámica del Cenote Sagrado. Reporte presentado a FAMSI No. 9761, (www.famsi.org/reports/9761 es/index.htlm)

Pool Cab, Marcos

1997 "Crecimiento de una unidad doméstica". Tesis de licenciatura en Ciencias Antropológicas con especialidad en Arqueología, México: Universidad Autónoma de Yucatán.

Quiñones Cetina, Lucía

1998 Resumen de los complejos de la secuencia cerámica de Izamal. Informe dirigido al CRY-INAH. Mecanografiado. México: INAH. 
Quiñones Cetina, Lucía

2003 "Del Preclásico Medio al Clásico Temprano: una propuesta de fechamiento para el área nuclear de Izamal, Yucatán". Tesis de licenciatura en Ciencias Antropológicas con especialidad en Arqueología, México: Universidad Autónoma de Yucatán.

Robles Castellanos, Fernando

1990 La secuencia cerámica de la región de Cobá, Quintana Roo. México: INAH (Colección Científica, 184).

Simmons, Michael

1980 The archaeological ceramics of Dzibilchaltún, Yuc. Mecanografiado. México: Biblioteca CRY-INAH.

Smith, Robert E.

1971 The pottery of Mayapán. Cambridge: Harvard University (Pappers of the Peabody Museum on Archaeology and Etnology, 66).

Varela Torrecillas, Carmen

1994 "El clásico medio en el noroccidente de Yucatán: la fase Oxkintok Regional en Oxkintok (Yucatán) como paradigma". Tesis de doctorado en Arqueología maya, Madrid: Universidad Complutense.

Willey, G., T. P. Culbert y R. E. W. Adams

1967 "Maya Lowland ceramics: a report from 1965 Guatemala City Conference", American Antiquity, 32 (3): 289-325. Salt Lake City: Society for American Archaeology. 